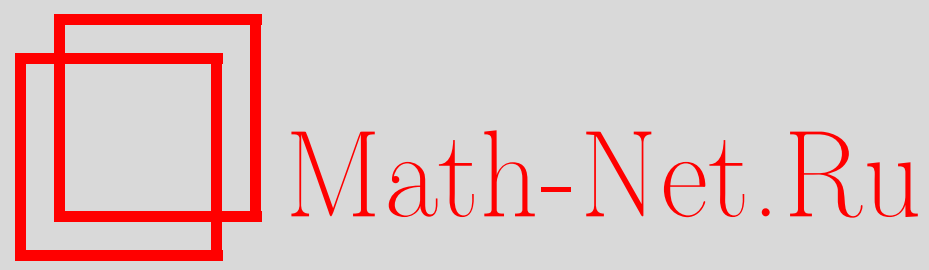

В. И. Рыбаков, О сходимости на границе единичного шара сопряженного пространства, Матем. заметки, 1996, том 59, выпуск 5, 753-758

DOI: https://doi.org/10.4213/mzm1769

Использование Общероссийского математического портала MathNet.Ru подразумевает, что вы прочитали и согласны с пользовательским соглашением

http://www . mathnet.ru/rus/agreement

Параметры загрузки:

IP : 18.209 .158 .208

26 апреля 2023 г., 13:27:57

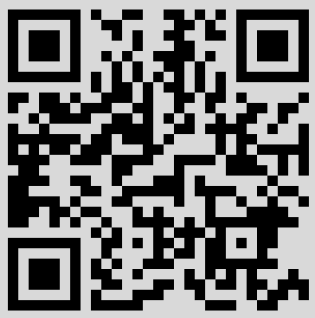


том 59 выпуСК 5 май 1996

\section{О СХОДИМОСТИ НА ГРАНИЦЕ ЕДИНИЧНОГО ШАРА СОПРЯЖЕННОГО ПРОСТРАНСТВА}

\section{В. И. Рыбаков}

Различньпи авторами ранее было установлено наличие некоторых свойств у банаховых пространств при вьполнимости определенных требований к множеству крайних точек единичного шара сопряженного пространства. В заметке устанавливается наличие этих свойств при выполнимости требований к меньшему (чем семейство крайних точек) множеству, а именно, требований к множеству точек какой-либо границы единичного шара. Перед формулировками утверждений указьваем источники, где имеются результаты, усиление которых предлагается.

Все встречающиеся топологические пространства предполагаются отделимьпи. Если $X$ - линейное нормированное пространство, то сопряженное к нему обозначаем $X^{*}$ (рассматриваем линейные пространства над $\mathbb{R}$ и, не оговаривая особо, бесконечномерные); для $x \in X, x^{*} \in X^{*}$ значение $x^{*}$ на $x$ обозначаем через $\left\langle x^{*}, x\right\rangle$ или через $x^{*}(x)$. Если $S$ какое-либо множество, а $F$ - семейство функций на нем, то $\sigma(S, F)$ - слабейшая из топологий в $S$, при которых все функции из $F$ непрерывны на $S$. Топологию $\sigma\left(X^{*}, X\right)$ на $X^{*}$ (или на каком-либо подмножестве $X^{*}$ ) обозначаем через $w^{*}$, а топологию $\sigma\left(X, X^{*}\right)$ на $X$ - через $w$ (назьвая ее слабой). Топологическое пространство $K$ называют счетно компактным, если каждое его бесконечное подмножество имеет в $K$ предельную точку. Если $(S, t)$ - топологическое пространство и $E \subset S$, то топологию, индуцированную на $E$ из $(S, t)$, будем также обозначать через $t$, рассматривая топологическое пространство $(E, t)$.

Для нормированного пространства $X$ пусть $B(X)$ - замкнутьй единичньй шар пространства $X, S(X)$ - его единичная сфера (т.е. $S(X)=\{x \in X:\|x\|=1\}) ;$ для $A \subset X$ через [A] обозначаем замкнутое линейное подпространство в $X$, порожденное $A$, а через $\overline{c o} A$ - замкнутую в $X$ вьпуклую оболочку $A$. Если $K$ - подмножество в $X^{*}$, то через $\Gamma(K)$ условимся обозначать какую-либо гранищу $K$ (если она существует), т.е. такое подмножество в $K$, что для любого $x \in X$ найдется $x_{0}^{*} \in \Gamma(K)$, для которого $\left\langle x_{0}^{*}, x\right\rangle=\sup \left\{x^{*}(x), x^{*} \in K\right\}$. Например, если $K$ $w^{*}$-компактное выпуклое множество в $X^{*}$, то множество крайних точек

Математические заметки, т. 59, в. 5

(C) В. И. Рываков 1996 
$K$ является $\Gamma(K)$, т.е. границей $K$.

Пусть $X$ - банахово пространство и $E$ - семейство крайних точек множества $B\left(X^{*}\right)$. Обобщая один из результатов Гротендика, в [1] и в [2] было установлено, что всякое ограниченное в $X$ множество, являюшееся счетно компактным в топологии $\sigma(X, E)$, будет слабо компактньм в $X$. Нижеследующее утверждение усиливает этот результат работ [1], [2].

Tеорема 1. Пусть $X$ - банахово пространство $и \Gamma=\Gamma\left(B\left(X^{*}\right)\right)$. Если $K$ ограничено в $X$ и пространство $(K, \sigma(X, \Gamma))$ счетно компактно, то $K$ слабо компактно в $X$.

ДоКАЗАТЕЛЬСТво. Нетрудно проверить, что пространство $(X, \sigma(X, \Gamma))$, и тем более $(K, \sigma(X, \Gamma))$, отделимо. Для доказательства теоремы достаточно установить, что всякая последовательность из $K$ содержит слабо сходящуюся подпоследовательность. Возьмем произвольную последовательность $\left(x_{n}\right) \subset K$ (можно считать, что все ее члены попарно различны, так что множество $\left\{x_{n}, n \in \mathbb{N}\right\}$ бесконечно) и пусть $Y=\left[x_{n}, n \in \mathbb{N}\right]$, а $K_{1}$ - замыкание множества $\left\{x_{n}, n \in \mathbb{N}\right\}$ в топологическом пространстве $(K, \sigma(X, \Gamma))$. Из условия теоремы и свойства сохранения счетной компактности при переходе к замкнутому подмножеству, заключаем: $K_{1}=\left(K_{1}, \sigma(X, \Gamma)\right)$ счетно компактно. Обозначим через $\varphi$ отображение, являющееся сужением элементов из $B\left(X^{*}\right)$ на $Y$ (т.е. $\varphi: B\left(X^{*}\right) \rightarrow Y^{*}$ ), и пусть $T=\varphi(\Gamma)$. Всякий $x \in K_{1}$ определяет на $T$ функцию $f_{x}$, именно: для $t \in T \quad f_{x}(t)=\left\langle x, x_{t}^{*}\right\rangle$, где $x_{t}^{*} \in \Gamma$ и $t=\varphi\left(x_{t}^{*}\right)$.

Сначала установим, что из $\left(x_{n}\right)$ можно выделить подпоследовательность, сходящуюся в каждой точке из $T$. Предположим противное. Тогда существует подпоследовательность $\left(x_{n}^{\prime}\right)$ последовательности $\left(x_{n}\right)$, непустое счетное подмножество $L \subset T$ и вещественные числа $r, \delta$ с $\delta>0$, так что для любого открытого $U$ в $\left(B\left(Y^{*}\right), w^{*}\right)$ c $U \cap L \neq \varnothing$ найдутся $t_{1}, t_{2} \in U \cap L$ c $f\left(t_{1}\right)<r$ и $f\left(t_{2}\right)>r+\delta$, где $f(t)=\lim _{n}\left\langle x_{n}^{\prime}, t\right\rangle, t \in L[3$, теорема 3]. Пусть $z \in K_{1}$ предельная точка множества $\left\{x_{n}^{\prime}, n \in \mathbb{N}\right\}$ и $Z=[z \cup Y]$. $Z$ cепарабельно, а $z$-непрерывная функция на компактном множестве $\left(B\left(Z^{*}\right), w^{*}\right)$. Если $T_{1}$ - совокупность сужений элементов из $\Gamma$ на $Z$, а $\varphi_{1}$ - отображение, являющееся сужением элементов из $T_{1}$ на $Y$ (тогда $\varphi_{1}\left(T_{1}\right)=\varphi(\Gamma)$ ), то для каждого $t \in L$ выберем элемент $v_{t} \in T_{1}$, для которого $\varphi_{1}\left(v_{t}\right)=t$. Положим $V=\left\{v_{t}, t \in L\right\}$, очевидно, $z\left(v_{t}\right)=f(t), t \in L$. Обозначим

$$
\begin{gathered}
A_{n}=\left\{v \in V: x_{n}^{\prime}(v)<r\right\} \cap\{v: z(v)<r\}, \\
B_{n}=\left\{v \in V: x_{n}^{\prime}(v)>r+\delta\right\} \cap\{v: z(v)>r+\delta\} .
\end{gathered}
$$

Из построения $V, L$ и свойств функций $x_{n}^{\prime}, z, f$ имеем: существует такое бесконечное подмножество $\mathbb{N}_{1}$ в $\mathbb{N}$, что для каждого $n \in \mathbb{N}_{1}$

$$
\bigcap\left\{\varepsilon_{i} B_{i}: i \in \mathbb{N}_{1}, i \leqslant n\right\} \neq \varnothing
$$


при любом выборе $\varepsilon_{i}$, принимающих значения 0 или 1 , где $0 \cdot B_{i}=A_{i}$, $1 \cdot B_{i}=B_{i}$, (свойство $(*)$ можно установить индукцией по $\left.n\right)$. Если $\bar{A}_{i}, \bar{B}_{i}$ - замыкание в $\left(B\left(Z^{*}\right), w^{*}\right)$ соответственно $A_{i}, B_{i}$, то для всякого $\Delta=\left(\varepsilon_{i}\right) \in\{0 ; 1\}^{\mathbb{N}_{1}}$ существует $z_{\Delta}^{*} \in \bigcap_{i \in \mathbb{N}_{1}} \varepsilon_{i} \bar{B}_{i}$, где $0 \cdot \bar{B}_{i}=\bar{A}_{i}$, $1 \cdot \bar{B}_{i}=\bar{A}_{i}$; при этом для $\Delta_{1}, \Delta_{2} \in\{0 ; 1\}^{\mathbb{N}_{1}}, \Delta_{1} \neq \Delta_{2}$, выполняется неравенство $\left|z\left(z_{\Delta_{1}}^{*}\right)-z\left(z_{\Delta_{2}}^{*}\right)\right| \geqslant \delta$. В таком случае метризуемое пространство $\left(B\left(Z^{*}\right), w^{*}\right)$ (расстояние $\rho$ можно определить, например, равенством

$$
\left.\rho\left(z_{1}^{*}, z_{2}^{*}\right)=\left|z\left(z_{1}^{*}\right)-z\left(z_{2}^{*}\right)\right|+\sum_{n=1}^{\infty} \frac{1}{2^{n}}\left|x_{n}\left(z_{1}^{*}\right)-x_{n}\left(z_{2}^{*}\right)\right|, \quad z_{1}^{*}, z_{2}^{*} \in B\left(Z^{*}\right)\right)
$$

несепарабельно, что противоречит компактности $\left(B\left(Z^{*}\right), w^{*}\right)$. Это доказывает, что из последовательности $\left(x_{n}\right)$ можно выделить подпоследовательность $\left(y_{n}\right)$, сходящуюся на $T$.

Итак, для всякого $t \in T$ существует $\lim _{n} y_{n}(t)$, тогда существует $\lim _{n} y_{n}\left(x^{*}\right)$ для любого $x^{*} \in \Gamma$, т.е. $\left(y_{n}\right)$ является последовательностью Коши в пространстве $K_{1}$. Вследствие счетной компактности $K_{1}$, найдется элемент $y \in K_{1}$, являющийся предельной точкой для $\left\{y_{n}, n \in \mathbb{N}\right\}$, а так как $\left(y_{n}\right)$ - последовательность Коши в $K_{1}$, то $\left(y_{n}\right)$ сходится к $y$ в $K_{1}$. Следовательно, $\lim _{n} y_{n}\left(x^{*}\right)=y\left(x^{*}\right)$ при любом $x^{*} \in \Gamma$. Согласно [4, следствие 11], $\left(y_{n}\right)$ слабо сходится в $X$ к $y \in K_{1} \subset K$. Это доказывает теорему.

Нижеследующее утверждение является усилением результатов, полученных в [5, теорема 9], [6, III.3]. Напомним некоторыепонятия и обозначения. Топологическое пространство $T$ назьвают счетно определенньм, если существует такое компактное пространство $K$, содержащее $T$ в качестве подпространства, и такое счетное семейство компактов $\left\{F_{n}, n \in \mathbb{N}\right\}$ в $K$, что для всякого $t \in T \bigcap\left\{F_{n}, t \in F_{n}\right\}$ содержится в $T$ [7], [8, IV.9.2]. Если $X$ - нормированное пространство и $E$ счетно определенное подпространство в $(E, w)$, то говорим, что $E$ слабо счетно определено в $X$. Для топологического пространства $E$ через $C_{p}(E)$ обозначаем пространство всех вешественных непрерывных функций на $E$, наделенное топологией поточечной сходимости на $E$. Определение пространства Асплунда см., например, в [9].

Tеорема 2. Пусть $X-$ банахово пространство, $K-w^{*}$-компактное выпуклое множество в $X^{*}$ и $\Gamma(K)$ содержится в слабо счетно определенном подмножестве пространства $X^{*}$. Тогда $K=\overline{\mathrm{co}} \Gamma(K)$. Если при этом $K=B\left(X^{*}\right)$, то $X$ - пространство Асплунда. 
ДоКАЗАТЕЛЬСТВо. Пусть $E$ - слабо счетно определенное в $X^{*}$ множество, для которого $\Gamma(K) \subset E$. Множество $B\left(X^{* *}\right)$ можно рассматривать как подмножество $F$ в $C_{p}(E)$ (с отждествлением некоторых элементов из $B\left(X^{* *}\right)$, если точки из $E$ не разделяют элементы из $\left.B\left(X^{* *}\right)\right)$. В пространстве $C_{p}(E)$ множество $F$ является компактом типа $\mathscr{E}_{2}$ (三 компакты Гулько) $[7,3.7]$. Напомним, что в компакте типа $\mathscr{E}_{2}$ всякая точка, принадлежащая замыканию какого-либо множества, является пределом последовательности точек этого множества (см. [7, 6.4] либо [8, III.6.2]). Тогда равенство $K=\overline{\mathrm{co}} \Gamma(K)$ следует из $[6,1.2]$.

Если $K=B\left(X^{*}\right)$, то (так как $\overline{c o} \Gamma(K)=B\left(X^{*}\right)$ и $\left.\Gamma(K) \subset E\right)$ замыкание в $X^{*}$ линейной оболочки слабо счетно определенного множества $E$ совпадает с $X^{*}$. В таком случае $X^{*}$ - слабо счетно определенное (или WCD) банахово пространство (см. [7, 3.6] либо [8, IV.2.10]). Тогда $X^{*}$ обладает свойством Радона-Никодима [7, комментарии после теоремы 6.1] и, следовательно, $X$ - пространство Асплунда [9, с. 210].

Ниже (теорема 3 ) будет получено усиление результата [10, следствие теоремы 1] и в качестве следствий получим утверждения, являющиеся развитием известных результатов (например, [11, теорема 1]).

Последовательность $\left(x_{n}, n \in \mathbb{N}\right)$ эелементов банахова пространства $X$ назьвается базисной, если она является базисом в $\left[x_{n}, n \in \mathbb{N}\right.$. Условие, что нормированное пространство $Y$ изоморфно подпространству пространства $X$, записываем $Y \hookrightarrow X$.

ЛЕмма. Пусть последовательность $\left(x_{n}\right)$ ограничена в банаховом пространстве $X$, отграничена от нуля (т.е. $\inf \left\{\left\|x_{n}\right\|, n \in \mathbb{N}\right\}>0$ ), $u \Gamma=\Gamma\left(B\left(X^{*}\right)\right)$. Если $x_{n} \rightarrow 0$ в $(X, \sigma(X, \Gamma))$, то из $\left(x_{n}\right)$ можсно выделить базисную подпоследовательность.

ДОКАЗАТЕЛЬСТВО. Ввиду $\sigma(X, \Gamma)$-сходимости ограниченной последовательности $\left(x_{n}\right)$, из [4, следствие 1] выводим, что $x_{n} \rightarrow 0$ в $(X, w)$ (т.е. $\left(x_{n}\right)$ слабо сходится к нулю). Тогда, согласно $[12$, с. 42$]$, из $\left(x_{n}\right)$ можно выделить базисную подпоследовательность.

Теорема 3. Для того, чтобы банахово пространство $X$ не содержало подпространства, изоморфного с $c_{0}$, необходимо и достаточно, чтобы всякий ряд $\sum_{n} x_{n}$, для которого

$$
\sum_{n}\left\langle x^{*}, x_{n}\right\rangle \mid<\infty, \quad x^{*} \in \Gamma=\Gamma\left(B\left(X^{*}\right)\right)
$$

был сходящимся.

ДокАЗАТЕЛЬСтво. Если существует изоморфноевложение $j: c_{0} \rightarrow X$, то ряд $\sum_{n} j\left(u_{n}\right)$, где $\left(u_{n}\right)$ - естественньй базис в $c_{0}$, будет обладать свойством, что $\sum\left|\left\langle j\left(u_{n}\right), x^{*}\right\rangle\right|<\infty$ для всякого $x^{*} \in X^{*}$ (а не только для 
$x^{*}$ из какой-либо гранищы множества $\left.B\left(X^{*}\right)\right)$, однако, этот ряд не будет сходящимся.

Докажем обратное утверждение. Пусть существует ряд $\sum_{n} x_{n}$, $x_{n} \in X$, не являющийся сходящимся, но такой, что (1) выполнено. Установим, что $c_{0} \hookrightarrow X$. Можно считать, что $\left\|x_{n}\right\|=1, n \in \mathbb{N}$. Действительно, из условия следует: найдутся такое $\varepsilon>0$ и такие натуральные числа $k_{n}, p_{n}\left(k_{n}+p_{n}<k_{n+1}\right)$, что $z_{n}=\sum_{i=k_{n}}^{k_{n}+p_{n}} x_{i}$ удовлетворяют неравенству $\left\|z_{n}\right\|>\varepsilon, n \in \mathbb{N}$; если положим $y_{n}=z_{n} /\left\|z_{n}\right\|$, то $\left\|y_{n}\right\|=1$ и $\sum\left|\left\langle x^{*}, y_{n}\right\rangle\right|<\infty, x^{*} \in \Gamma$.

Из условия (1) вьводим: для каждого $x^{*} \in \Gamma\left\langle x^{*}, x_{n}\right\rangle \rightarrow 0$ при $n \rightarrow \infty$. Выделим из $\left(x_{n}\right)$ базисную подпоследовательность (согласно лемме), которую обозначим через $\left(y_{n}\right)$. Пусть $Y=\left[y_{n}, n \in \mathbb{N}\right\}$. $\left(y_{n}\right)$ является базисом в $Y$, тогда (так как $\left.y_{n} \in S(X)\right)$ если $y=\sum_{n} \alpha_{n} y_{n}$, то $\left(\alpha_{n}\right) \in c_{0}$ для каждого $y \in Y$.

Определим отображение $T: Y \rightarrow c_{0}$ условием: если $y=\sum \alpha_{n} y_{n} \in Y$, то $T y=\left(\alpha_{n}\right) . T-$ линейное вложение. Если обратное отображение $T^{-1}$ непрерьвно, то $T Y=c_{0}$ и $T$ является изоморфизмом, значит $Y$ - подпространство в $X$, изоморфное $c_{0}$.

Пусть $T^{-1}$ не является ограниченным. Рассмотрим сопряженное к $T$ отображение $T^{*}: \ell_{1} \rightarrow Y^{*}$ и обозначим через $\left(e_{n}\right)$ естественньй базис в $\ell_{1}$. Положим $\varphi: X^{*} \rightarrow Y^{*}$ сужение элементов из $X^{*}$ на $Y$, тогда $\varphi(\Gamma)-$ гранища для $B\left(Y^{*}\right)$. Если $y^{*} \in \varphi(\Gamma)$ и $\left\langle y^{*}, y_{n}\right\rangle=\gamma_{n}$, то, ввиду (1), $\sum\left|\gamma_{n}\right|<\infty$. Рассматривая $v=\left(\gamma_{n}\right)$ как эелемент в $\ell_{1}$, вследствие $T^{*} v \in Y^{*}$ и $\left\langle T^{*} v, y_{n}\right\rangle=\left\langle v, T y_{n}\right\rangle=\gamma_{n}$, заключаем: $y^{*}=T^{*} v$ (ибо точки $\left\{y_{n}, n \in \mathbb{N}\right\}$ разделяют элементы из $\left.Y^{*}\right)$. Итак, $T^{*} \ell_{1}$ содержит множество $\varphi(\Gamma)$, являющееся гранищей для $B\left(Y^{*}\right)$. Тогда $c_{0} \hookrightarrow Y[13$, теорема 2], следовательно, $c_{0} \hookrightarrow X$. Теорема доказана.

Подмножество $E \subset B\left(X^{*}\right)$ называется нормирующим, если

$$
\inf \left\{\sup \left\{\left|x^{*}(x)\right|, x^{*} \in E\right\}, x \in S(X)\right\}>0 \text {. }
$$

СлЕДСТВИЕ 1. Пусть $X-$ банахово пространство. Если $\Gamma=\Gamma\left(B\left(X^{*}\right)\right)$ может быть покрыто счетным числом $\left\{H_{n}\right\}$ ненормирующих множеств $\left(H_{1} \subset H_{2} \subset \ldots\right)$, то $c_{0} \hookrightarrow X$.

ДокАЗАТЕЛЬСТво. Для каждого $n \in \mathbb{N}$ найдется $x_{n} \in S(X)$ такой, что $\sup \left\{\left|x^{*}\left(x_{n}\right)\right|, x^{*} \in H_{n}\right\}<1 / 2^{n}$. Тогда (так как для любого $x^{*} \in \Gamma$ существует номер $n_{0}$, что $x^{*} \in H_{n}$ при $\left.n \geqslant n_{0}\right) \sum\left|\left\langle x^{*}, x_{n}\right\rangle\right|<\infty$ при любом $x^{*} \in \Gamma$. Из теоремы 3 заключаем: $c_{0} \hookrightarrow X$.

СлЕДСТВИЕ 2. Пусть $X$ - банахово пространство. Если $\Gamma=\Gamma\left(B\left(X^{*}\right)\right)$ может быть покрыто счетным числом компактных мнохсеств, то всякое замкнутое линейное подпространство в $X$ содержит подпространство, изоморфное $c_{0}$. 
ДокаЗАТЕЛЬСтво. Сначала установим, что если $Z$ - нормированное пространство (бесконечномерное) и $K$ - компактное в $B\left(Z^{*}\right)$ множество, то $K$ не является нормирующим. Действительно, в противном случае $w^{*}$-замыкание множества $F=\overline{\mathrm{co}}(-K \cup K)$ содержало бы шар $\left\{x^{*} \in X^{*}:\left\|x^{*}\right\| \leqslant r\right\}$ для некоторого $r>0$. Множество $F$, являясь компактным в $Z^{*}$, будет и $w^{*}$-компактным и, следовательно, $w^{*}$-замкнуто. Тогда пространство $Z^{*}$ имеет компактную окрестность нуля, что возможно лишь в случае, когда $Z^{*}$ конечномерно, чего по условию нет. Итак, $K$ - ненормирующее множество.

Пусть $K_{n}, n \in \mathbb{N}$, компактные в $X^{*}$ множества такие, что $\Gamma \subset \bigcup_{n} K_{n}$ и $Y$ - линейное замкнутое бесконечномерное подпространство пространства $X$. Если $\varphi: X^{*} \rightarrow Y^{*}$ - отображение сужения, то множество $\varphi(\Gamma)$ является границей для $B\left(Y^{*}\right)$, множества $F_{n}=\varphi\left(K_{n}\right)$ - компактны в $Y^{*}$ и $\varphi(\Gamma) \subset \bigcup_{n} F_{n}$. Из предыдущей части доказательства и следствия 1 заключаем: $c_{0} \hookrightarrow Y$.

Тульский государственный

Поступило

педагогический университет 10.05 .94

\section{СПИСОК ЦИТИРОВАННОЙ ЛИТЕРАТУРЫ}

[1] Bourgain J., Talagrand M. Compacite extremale // Proc. Amer. Math. Soc. 1980 . V. 80. P. 68-70.

[2] Khurana S.S. Point-wise compactness on extreme points // Proc. Amer. Math. Soc. 1981. V. 83. № 2. P. 347-348.

[3] Rosenthal H. Point-wise compact subsets of the first Baire class, with some applications to Banach space theory // Var. Publ. Ser. Math. Inst. Aarhus Univ. 1975. № 24. P. 176-187.

[4] Simons S. A convergence theorem with boundary // Pacific J. Math. 1972. V. 40. P. 703-708.

[5] Saab E. Extreme points, separability and weak $K$-analiticity in dual Banach spaces // J. London Math. Soc. 1981. V. 23. P. 165-170.

[6] Grodefroy G. Boundaries of a convex set and interpolation sets // Math. Ann. 1987. V. 277. P. 173-184.

[7] Talagrand M. Espaces de Banach faiblement $K$-analitiques // Ann. Math. 1979. V. 110. № 3. P. 407-438.

[8] Архангельский А. В. Топологические пространства функций. М.: Изд-во МГУ, 1989.

[9] Giles J. R. Convex analysis with application in the differentiation of convex functions. London: Pitman, 1982.

[10] Elton J. Extremely weakly unconditionally convergent series // Israel J. Math. 1981. V. 40. № 3, 4. P. 255-258.

[11] Фонф В. П. Слабо экстремальные свойства банаховых пространств // Матем. заметки. 1989. Т. 45. №6. С. 83-91.

[12] Diestel J. Sequences and series in Banach spaces. New York: Springer Verlag, 1984.

[13] Фонф В. П. Множества суперпервой категории в банаховых пространствах // Функцион. анализ и его прилож. 1991. Т. 25. № 4. С. 66-69. 